\title{
Conducting competency-based assessments during the COVID-19 pandemic: Moving to remote assessments
}

\author{
A. Cooper*, J. Thompson \\ Keele University, United Kingdom
}

\section{Keywords}

Competency-based Assessment

Competence

Pharmacy Student

Remote Assessment

United Kingdom

\section{${ }^{*}$ Corresponding author}

a.cooper1@keele.ac.uk

Summary: At Keele University School of Pharmacy and Bioengineering, all Master of
Pharmacy students undertake Competency Based Assessments (CBAs) towards the end
of each academic year. CBAs simulate 'real life' settings in pharmacy and assess a
student's ability to apply their knowledge, skills and understanding in a safe simulated
environment. As a result of the COVID-19 pandemic the assessments could not be
conducted in the usual way. Instead, they were re-designed to be carried out remotely
using Google Meet software, to ensure students were still meeting the General
Pharmaceutical Council outcomes for education and training. With a strong team who
had previous experience in the design and delivery of CBAs, the delivery of remote
CBAs was successful and enabled students' competence to be measured virtually. More
time and preparation will enable greater understanding as to how virtual assessments
can fit into a primarily non-virtual degree.

Summary: At Keele University School of Pharmacy and Bioengineering, all Master of Pharmacy students undertake Competency Based Assessments (CBAs) towards the end of each academic year. CBAs simulate 'real life' settings in pharmacy and assess a environment. As a result of the COVID-19 pandemic the assessments could not be conducted in the usual way. Instead, they were re-designed to be carried out remotely using Google Meet software, to ensure students were still meeting the General Pharmaceutical Council outcomes for education and training. With a strong team who had previous experience in the design and delivery of CBAs, the delivery of remote time and preparation will enable greater understanding as to how virtual assessments can fit into a primarily non-virtual degree.

\section{Background and Context}

The role of the pharmacist has evolved, moving from predominantly that of dispensing medicines and provision of information to patients and healthcare professionals, to playing a pivotal role in the delivery of clinical services, management of chronic conditions, lifestyle-related diseases and prevention and detection of ill health (Department of Health, 2016; Department of Health and Social Care, 2019).

At Keele University School of Pharmacy and Bioengineering, students in each year of the Master of Pharmacy (M.Pharm.) degree must undertake Competency Based Assessments (CBAs). These are designed to assess students' competency over a range of learning outcomes in preparation for wider pharmacy roles.

The outcome levels in Standard Ten of the General Pharmaceutical Council's (GPhC) Standards for Education and Training have been derived using Miller's triangle (Miller, 1990; GPhC, 2011). CBAs simulate a 'real life' setting, enabling students to 'show how' they can apply their knowledge, skills and understanding of the course content in a safe environment (Figure A).
The CBAs run at Keele University are designed so that each student undertakes six stations, and complexity increases as students' progress through the course. Students have sixminutes to complete each station with reading time prior. Stations include clinical checks, counselling and managing interactions. As a result of the COVID-19 pandemic, face-toface assessments were cancelled. Instead, CBAs were conducted virtually to ensure students were still meeting the GPhC outcomes. This case study will discuss the development and running of the Stage two M.Pharm. students' CBAs via Google Meet.

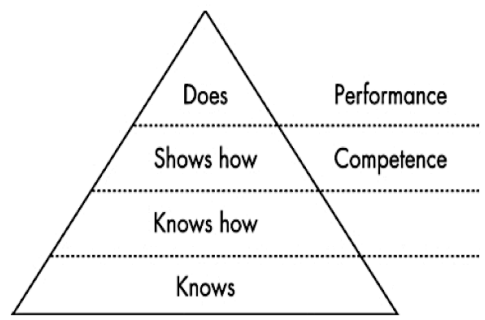

Figure A: Miller's triangle. CBAs allow students reach the 'Shows how' level of Miller's triangle (Carr, 2004) 


\section{Educational Description}

The CBAs were designed to run virtually using Google Meet as this would allow linking with University staff and students via Google accounts. The virtual running of the CBA led to a change in the stations which students would sit. It was not feasible to run the more 'practical' stations such as accuracy checking. Students would sit four stations in total; three 'face-to-face' stations being conducted virtually on Google Meet and one written station created as a Google Form. The 'face-to-face' CBA stations were interaction check, clinical check, and patient counselling. Students had eight minutes to complete each station. The written station surrounded the legality of controlled drugs. The written station was uploaded onto the Keele Learning Environment (KLE) and opened for a one-hour period the day after the 'face-to-face' stations, ensuring those in different time zones would be able to access it.

Nine sets of stations were created; each 'set' consisted of the three 'face-to-face' stations and the one written station. Newly written stations were reviewed by external examiners. CBAs were previously marked on tablet devices using an app created by a team at Keele University's School of Medicine. These devices were not accessible from home, instead Google Forms were created with analytical checklists for each 'face-to-face' station. This allowed assessors to mark each student 'yes' or 'no' on the station components and provide typed feedback for the students to receive at a later date. Sixteen assessors were recruited to assess the students in the 'face-to-face' stations. All assessors were GPhC registered academic staff within the School of Pharmacy. Assessor training was held on Google Meet.

In advance of the assessment, an announcement was released on the KLE to inform the students of the virtual CBA. All students were invited to IT drop-in sessions via Google Meet two days prior to the assessment to resolve any potential IT problems. Contingency planning included using audio only if no webcam was available and utilising the chat function on Google Meet where necessary.

On the assessment day, a Microsoft Teams group was created for the assessors and the CBA development team. This allowed for queries to be answered immediately, conversation between assessors, and helped to ensure quality assurance.

Each assessor virtually assessed eight students. Pre-arranged 45-minute time-slots were created for each student, accounting for any time zone requirements or other circumstances, such as childcare. At the start of each virtual call, the student's name and email address was confirmed. The set of stations was then emailed over to the student. Upon confirmation that they had received and opened the document, the assessment began. Each student completed their three 'face-to-face' stations with the same assessor. All students in a particular time-slot received the same set of stations. The students in the next time-slot would then receive the next set of stations, and so on.
The main avenue of feedback was anecdotal via the Microsoft Teams chat, one-to-one chats with assessors and through staff meetings. Anecdotal feedback was also received from students.

\section{Outcomes and Recommendations}

Both the 'face-to-face' and written stations were successful in their running. The assessment demonstrated the ability to measure student competence in a virtual setting. The overall student performance was comparable to in person CBAs. Google Meet was an effective tool to assess students in the 'face-to-face' stations. All students and staff were able to access it. Few technological problems were reported, but where there were issues, the contingency plans ensured all students could undertake their assessment. Any universities considering a similar online assessment should have a strong team with previous experience in designing and delivering CBAs. Having IT support prior to and on the assessment day was also pivotal to success. Full preparation was key to the smooth running of the day.

Care needs to be taken to ensure students who have been assessed earlier in the day do not share the material with other students. Using different 'sets' of stations helped prevent possible cheating. There was evidence of collusion during the assessment day; it had not be explicitly stated that the material sent to the students for the assessment was confidential, however it was expected that M.Pharm. students would understand this. Moving forward, a declaration will be created for students to sign prior to the assessment specifying the confidential nature of the material and setting out the consequences if the declaration is broken. Both the assessors and the students reported feeling relaxed for the virtual CBA. Assessor training was successful at easing any anxiety prior to the day. The students valued the level of information they were provided and the IT drop-ins prior to the assessment ensured they felt more confident.

This approach to CBAs will be continued in the future. With more time, considerations as to how the 'practical' stations can be integrated into a virtual setting will be key to understanding how virtual assessments can fit into a primarily non-virtual degree.

\section{References}

Carr, S.J. (2004) Assessing clinical competency in medical senior house officers: how and why should we do it? Postgraduate Medical Journal, 80, 63-66. http://dx.doi.org/10.1136/pmi.2003.011718

Department of Health. (2016). Operational Productivity and Performance in English NHS Acute Hospitals: Unwarranted Variations. Plus Media Solutions, London

Department of Health and Social Care. (2019). The Community Pharmacy Contractual Framework. GOV.UK, London

GPhC [General Pharmaceutical Council]. (2011). Future pharmacists: Standards for the initial education and training of pharmacists. General Pharmaceutical Council, London

Miller, G.E. (1990). The assessment of clinical skills/competence/ performance. Academic Medicine, 65(9 Suppl), S63-7. https:// dx.doi.org/10.1097/00001888-199009000-00045 